\title{
AGNs in Shakhbazian Compact Groups
}

\author{
A.S. Amirkhanian and A.G. Egikian \\ Byurakan Astrophysical Observatory, Byurakan, 378433, Armenia
}

\author{
H. Tiersch and D. Stoll
}

\section{Sternwarte Koenigsleiten, Muenchen, Germany}

The results of CCD spectroscopic observations of Shakhbazian compact groups of galaxies (SHCGs) with the 1.54-m (La Silla, Chile), 2.2-m (Calar Alto, Spain) and 2.6-m (Byurakan) telescopes are presented. According to these preliminary data, about $10 \%$ of member galaxies in SHCGs are emission-line galaxies (ELGs) including the broad-line AGNs (of classical Seyfert 1 type) and the narrow-emission-line galaxies.

A research program has been developed in the University of Potsdam, Potsdam Astrophysikalisches Institut in cooperation with other observatories (particularly with Byurakan Astrophysical Observatory) to perform photometric and spectroscopic investigations of galaxies in the SHCGs. Within the framework of this program the redshift (radial velocity) measurements have been carried out for more than 200 galaxies in 36 SHCGs. The MIDAS software package was used for processing and interpreting of the galaxy spectra. Most of these redshifts were measured for the first time. 180 member galaxies $(90 \%)$ in these groups have absorption spectra typical of $\mathrm{E}$ and SO galaxies. Twenty galaxies (10\%) turn out to be ELGs. They are in the range $0.02 \leq \mathrm{z} \leq 0.17$, i.e., the SHCGs lie in approximately the same redshift space as Abell clusters. These compact groups contain predominantly elliptical and lenticular galaxies (del Olmo 1988; Amirkhanian 1989) like the cores of rich, regular, centrally condensed clusters of galaxies. The fraction of spirals falls in the densest matter concentrations. On the other hand, it is a well-established fact that in the local universe the active objects tend to avoid the cores of dense clusters of galaxies (e.g. Green and Yee 1984). That is why the discovery of an emission-line population with broad-line AGNs in SHCGs (Tiersch et al. 1999) was unexpected. As shown by Dressler, Thompson and Shectman (1985) in their sample of 1268 galaxies in the feilds of 14 rich clusters the ELGs comprise $31 \%$ of the field galaxies but only $7 \%$ of the cluster galaxies. Similarly, according to their statistics AGNs make up 5\% of the field sample, but only $1 \%$ of the cluster sample. They note that the difference in the distribution of morphological types can only partially explain this effect. Obviously, some sort of environmental influence is present.

A cursory investigation in the literature of the frequency of ELGs in the Hickson compact groups (HCGs) (Shimada et al. 2000) and the south compact groups (SCGs) (Coziol et al. 2000) has resulted in conflicting data. Shimada et al. (2000) present results of their optical spectroscopy of 69 galaxies belonging to 31 HCGs of galaxies. They found that for each morphological type there is no statistically significant difference in the frequences of occurence of ELGs 
among the HCGs and in the field. The field galaxies data are taken from Ho, Filippenko and Sargent $(1995 ; 1997)$. But according to their statistics (Shimada et al. 2000) the difference for the total sample is significant, in that the ELGs (AGNs + HII nucleus galaxies) and the absorption galaxies are found in $70 \%$ and $30 \%$ of the HCG galaxies, respectively. Meanwhile, in the field the ELGs make up $89 \%$ and the absorption galaxies comprise only $11 \%$ of the sample.

The ELGs and the absorption galaxies comprise $73 \%$ and $27 \%$ of the SCGs galaxies, respectively (Coziol et al. 2000). This result is similar to that for HCGs. The population of galaxies in Shakhbazian groups differs strongly by morphological content. The frequency of emission-line galaxies in the HCGs and SCGs is much higher than in Shakhbazian groups. This difference is likely to be due to the following explanation. The great majority of Shakhbazian groups are at larger distances with respect to the HCGs, most of which are nearby systems at redshifts below 0.05 (Hickson et al. 1992). Moreover, extensive spectroscopic studies are needed to obtain the spectral activity types for all the galaxies in the 377 groups of the Shakhbazian sample (Shakhbazian 1973; Baier and Tiersch 1979 and references therein).

Finally we note that Shk $355 / 4$ is a classical Seyfert 1 galaxy at the same redshift as its host group with $z=0.0943$ (Tiersch et al. 1999). Moreover Shk $278 / 4$ is also a broad-line AGN in an early-type galaxy ( $\mathrm{z}=0.1205)$. This is the first active object in the SHCGs discovered by Spanish astronomers (del Olmo and Moles 1991). It has a remarkable resemblance to QSOs or QSO-like objects. At the same time, although more than $70 \%$ of the galaxies in SCGs probably have an active nucleus, there is no Seyfert 1 among 193 galaxies in 49 southern groups spectroscopically investigated by Coziol et al. (2000).

\section{References}

Amirkhanian, A. S. 1989, PhD. Thesis, Byurakan Astrophysical Observatory, Armenia

Baier, F. W., \& Tiersch, H. 1979, Astrofizika, 15, 33

Coziol, R., Iovino, A., \& Carvalho, R. R. 2000, AJ, 120,47

del Olmo, A. 1988, $\mathrm{PhD}$. Thesis, University of Granada, Spain

del Olmo, A., \& Moles, M. 1991, A \& A, 245,27

Dressler, A., Thompson, I. B. , \& Shectman, S. A. 1985, ApJ , 288,481

Green, R. F. , \& Yee, H. K. C. 1984, Ap.JS, 54,495

Ho, L. C., Filippenko, A. V., \& Sargent, W. L. W. 1995, ApJS, 98,477

Ho, L. C., Filippenko, A. V., \& Sargent, W. L. W. 1997, ApJS, 112, 315

Shakhbazian, R. K. 1973, Astrofizika, 9, 495

Shimada, M., Ohyama Y., Nishiura, S., Hurayama, T., \& Taniguichi Y. 2000, AJ, 119,2664

Tiersch, H., Stoll, D., Neizvestny, S., Amirkhanian, A. S., \& Egikian, A. G. 1999, in Active Galactic Nuclei and Related Phenomena, IAU Symp. 194, eds. Y. Terzian, D. Weedman, E. Khachikian, Astron. Soc. Pacif., Chelsea, Michigan, p. 394 